\title{
Wikigender: un outil novateur pour alimenter le débat sur l'égalité homme-femme
}

\author{
par Denis Drechsler
}

- Wikigender est le premier site Internet de I'OCDE utilisant la technologie « wiki »

- Ce site est le fer de lance de la nouvelle stratégie de communication de I'OCDE qui consiste à utiliser les outils web 2.0 pour atteindre un public nouveau

- L'implication du public dans le débat sur l'égalité homme-femme permettra d'alimenter et de soutenir les réformes dans ce domaine

Avez-vous déjà envisagé de comparer le nombre d'hommes et de femmes qui occupent un emploi salarié ? Dans quelle mesure exactement les femmes sont-elles moins bien payées que les hommes ? Qui sont les dirigeants d'entreprises, quelle est la proportion de personnes de sexe féminin parmi eux ? La parité homme-femme est-elle une réalité au sommet de l'État ?

Autant de questions qui méritent d'être posées, car la réponse à ces interrogations - ou à d'autres du même type détermine la manière dont les responsables politiques agissent pour favoriser le développement économique et social des femmes. Ces réponses nous informent également sur le degré d'efficacité des politiques visant à réduire les inégalités entre les sexes, ce qui n'est pas seulement fondamental au regard des droits humains, mais a aussi des implications très diverses sur la santé économique d'un pays.

\section{Statistiques sur l'égalité homme-femme : la grande inconnue}

Aussi étonnant que cela puisse paraître, on ne dispose que de très peu d'informations sur cette question à travers le monde. La rareté des données ventilées par sexe rend plus difficile la réalisation des Objectifs du millénaire pour le développement, qui consistent à parvenir à l'égalité entre les sexes en matière de scolarisation, de participation au marché du travail et de représentation politique. Lorsqu'ils existent, ces chiffres sont rarement comparables d'un pays à l'autre, et leur périodicité est trop irrégulière.

Souvent, les statistiques ventilées par sexe ne couvrent qu'un champ réduit : sélection d'indicateurs concernant le marché du travail, informations relatives à l'éducation et à la santé, émancipation économique des femmes. Les données chiffrées concernant d'autres formes de discrimination sexuelle, qui reposent par exemple sur des normes sociales restrictives ou des règles familiales, sont très rarement prises en compte par les offices nationaux de statistique et les organisations internationales. Ainsi, il n'existe pas de données internationales fiables relatives aux violences faites aux femmes, question pourtant centrale du débat sur l'égalité. À cet égard, la base de données Égalité homme-femme, Institutions et Développement (EID) du Centre de développement de I'OCDE est une exception notable.

\section{Wikigender fait son entrée}

La création d'une base de données exhaustive comme l'EID a représenté une avancée considérable dont la valeur est indéniable, mais cet outil présente un défaut inhérent à sa nature : les informations proviennent de sources officielles. Il était donc nécessaire d'aller plus loin. C'est là qu'intervient Wikigender (www.wikigender.org). Plus qu'un site Internet, le premier « wiki » de l'OCDE permet de s'adresser efficacement à la société civile et d'engager le dialogue sur l'égalité entre les sexes à tous les niveaux.

\section{Logo de Wikigender}

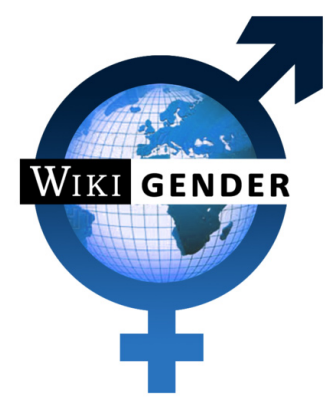


Wikigender est un forum ouvert pour partager et échanger des informations relatives à la situation des hommes et des femmes à travers le monde, permettant ainsi de discuter franchement des freins qui empêchent la concrétisation de l'égalité homme-femme. Le site, véritable base de données des savoirs, encourage également ses utilisateurs à participer activement à son contenu en formulant des commentaires, en participant à la rédaction de certains articles ou en créant de nouvelles entrées.

Cette initiative constitue la première - et la plus évidente manifestation de la nouvelle stratégie informatique de I'OCDE élaborée autour des outils web 2.0 tels que les « wikis ». Selon cette stratégie, I'OCDE doit jouer un rôle plus important d'accumulation et de transmission des connaissances dans un monde où les institutions comme les individus sont constamment bombardés d'information. L'organisation met au point une véritable démarche de gestion des connaissances en améliorant le classement de l'information, en réduisant les doublons et en facilitant le ciblage et la récupération des données.

Depuis son lancement en mars 2008, Wikigender a déjà constitué un répertoire riche de données et de statistiques couvrant des sujets aussi divers que la représentation politique des femmes au sein des institutions nationales, le rôle des hommes dans l'éducation des enfants et l'importance des femmes au regard du développement économique. De leur côté, les utilisateurs ont accès à plus de 350 articles et documents, dont des études détaillées par pays sur le thème de l'égalité entre les sexes provenant de pays membres ou non-membres de I'OCDE. Au cours de ses premiers mois de fonctionnement, le site a accueilli plus de 100000 visiteurs. Wikigender contient deux types de contenus différents, ce qui le distingue nettement des autres sites ayant recours à la technologie « wiki », telle la célèbre encyclopédie Wikipedia.
Le contenu fourni par les sources officielles (par exemple I'OCDE) est surligné et protégé. Les nouvelles informations qui sont saisies via la partie ouverte de Wikigender sont examinées par un groupe de plus de 300 spécialistes qui sont devenus des utilisateurs accrédités. Ils ont l'autorisation de suggérer des modifications du contenu ainsi que de créer ou de télécharger de nouveaux articles et documents. Il en résulte un filtrage systématique de l'intégralité du site permettant d'éliminer tout contenu à la fiabilité douteuse et de garantir un niveau de qualité maximal.

\section{Au-delà du partage des données \\ - Promouvoir un réel changement}

Wikigender ne se contente pas d'améliorer l'état des connaissances sur la situation des hommes et des femmes à travers le monde ; il vise également à sensibiliser l'ensemble de la population mondiale quant à la nature et à l'étendue des discriminations sexuelles, étape indispensable à l'éradication des préjugés et de la défiance qui font le lit de ces inégalités. Le fait d'impliquer les individus dans cette expérience d'apprentissage mutuel permettra de forger des alliances au niveau local avec des syndicats, des associations professionnelles et des enseignants, par exemple. Ces acteurs peuvent faire pression pour que les choses changent tout en mobilisant le grand public et en levant les peurs qui accompagnent inévitablement les réformes. Ce n'est qu'en s'assurant de l'implication réelle de l'ensemble de la société dans ce débat que nous pourrons comprendre ce qui fait obstacle à l'égalité entre les sexes et ainsi élaborer des politiques permettant de remédier à cette situation. Wikigender est une initiative d'importance cruciale pour la réussite de cette entreprise.

\section{Références :}

Wikigender en ligne à l'adresse www.wikigender.org
Les lecteurs sont invités à citer ou reproduire les informations des Repères du Centre de développement de I'OCDE dans leurs propres publications. En échange, le Centre demande les remerciements de rigueur ainsi qu'un exemplaire de la publication. Le texte intégral des Repères et d'autres informations sur le Centre de développement et ses travaux sont disponibles sur : www.oecd.org/dev
Centre de développement de I'OCDE 2, rue André-Pascal, 75775 Paris Cedex 16, France Tél : $33(0) 145.24 .82 .00$ Fax : $33(0) 144.30 .61 .49$ mél : cendev.contact@oecd.org 